\title{
AIDS em Crianças: Considerações Sobre a Transmissão Vertical
}

\author{
AIDS in Children: Observations on Vertical Transmission
}

Maria Beatriz Ortigão ${ }^{1}$

ORTIGÃO, M. B. AIDS in Children: Observations on Vertical Transmission. Cad. Saúde Públ., Rio de Janeiro, 11 (1): 142-148, Jan/Mar, 1995.

This paper seeks to review the epidemiological aspects of the acquired immunodeficiency syndrome (AIDS) in children and the vertical transmission of the human immunodeficiency virus (HIV). The modes of mother-to-infant transmission are discussed, including physiopathological aspects of HIV infection in utero, during, and/or after delivery.

Key words: AIDS; HIV; Infection; Children; Epidemiology; Vertical Transmission

\section{INTRODUÇÃO}

A Síndrome de Imunodeficiência Adquirida (AIDS) infantil foi reportada pela primeira vez ao Centro de Controle de Doenças (CDC Atlanta) em 1982, um ano após a descrição inicial da nova doença em adultos. De forma análoga ao que era observado nos adultos infectados pelo HIV, aquelas crianças apresentavam alterações da função imune, acarretando uma maior predisposição a infecções do que nos indivíduos-controles da mesma faixa etária. À medida que mais casos de crianças com sintomatologia semelhante foram surgindo, tornou-se evidente que tais pacientes apresentavam em comum mães infectadas pelo HIV. Desde então, a incidência de infecção pelo HIV em crianças vem aumentando em função da disseminação da infecção a mulheres em idade procriativa. Até o presente, como ainda não foram desenvolvidos tratamentos curativos nem uma vacina anti-HIV eficaz, a melhor estratégia para combater a infecção perinatal continua sendo a prevenção, o que torna fundamental a compreensão dos mecanismos de transmissão.

\footnotetext{
'Laboratório de Imunologia Clínica, Departamento de Imunologia, Instituto Oswaldo Cruz Fundação Oswaldo Cruz Avenida Brasil, 4365, Rio de Janeiro. RJ, 21045-900, Brasil.
}

\section{EPIDEMIOLOGIA}

Aproximadamente 75 a $80 \%$ das crianças portadoras do HIV são infectadas por transmissão vertical. Os demais 20 a $25 \%$ adquirem a infecção por transfusão de sangue ou derivados e, particularmente no caso de crianças maiores e adolescentes, por via sexual ou uso de drogas endovenosas. Este percentual tem sido verificado em diversos países (Kozinetz et al., 1990; Pape et al., 1983), especialmente naqueles, como os do continente africano, onde a transmissão heterossexual predomina (Ryder et al., 1989; Melbye et al., 1986). No Brasil, até julho de 1994 , verificou-se um total acumulado de 1718 casos de crianças com AIDS, dos quais 1101 (64\%) ocorreram por transmissão vertical (MS, 1993). A menor porcentagem de transmissão vertical observada no Brasil em relação a outros países pode dever-se ao fato de aqui considerarem-se casos pediátricos os indivíduos acometidos até 15 anos de idade, ao passo que nos outros países o limite de idade é de 13 anos. Devido a isto, aumenta-se proporcionalmente o número de adolescentes infectados por uso de drogas, por via sexual, ou hemofílicos.

Até julho de 1987, a transmissão vertical correspondia a $0,5 \%$ do total de casos de AIDS relatados no Brasil (MS, 1987). Ao final de 1992, a porcentagem havia atingido a faixa dos $2 \%$, mantendo-se nestes níveis segundo os dados 
preliminares de julho de 1994 (MS, 1994). Estes dados correlacionam-se com a maior incidência de infecção pelo HIV em mulheres, quer pelo uso de drogas injetáveis, quer por contato sexual com parceiros HIV positivos. Em 1987, 3,4\% do total de casos de AIDS ocorreu em mulheres acima de 15 anos enquanto que em 1993/94 o percentual foi de $26,6 \%$ (MS, 1994). A relação de casos homem/mulher que era de 10:1 em 1987 passa a ser de 3,75:1 em 1992 (MS, 1994). Este aumento pode estar relacionado à modificação do perfil global da transmissão do vírus que vem diminuindo em homossexuais e aumentando em drogadictos.

Inquéritos de soroprevalência realizados em mulheres grávidas no ano de 1989 demonstraram resultados variáveis em algumas cidades do Brasil. Em São Paulo, de 2857 grávidas testadas, foi observado $1 \%$ de positividade para anticorpos anti-HIV (Quinn et al., 1989), o mesmo ocorrendo em Salvador (MS, 1992). Já em Santos, cidade portuária onde há alta prevalência de drogadicção e prostituição, a soropositividade foi de 3,6\%. Embora o número de estudos em grávidas seja pequeno, estes dados podem predizer uma incidência cada vez maior de casos de AIDS pediátrica por transmissão materno-infantil.

\section{TRANSMISSÃO VERTICAL}

Ao nascimento, praticamente todas es crianças filhas de portadoras do vírus apresentam anticorpos anti-HIV séricos. Entretanto, a positividade sorológica deve-se à passagem de anticorpos maternos pela placenta e não à produção pelo lactente. Esta indefiniçãa diagnóstica pode persistir até os 15 meses de idade, época-limite em que se espera que os anticorpos maternos tenham sido substituídos pelos da criança, ou tenham negativado definitivamente.

Estudos epidemiológicos realizados em diversos países têm mostrado que 7 a 50\% das crianças filhas de mulheres HIV-positivas adquirem a infecção, a maioria concordando entre 20 e 30\% (Italian Multicentric Study, 1988; Blanche et al., 1989; Ryder et al., 1989; European Collaborative Study, 1991, 1992). As demais tornam-se negativas entre 12 e 15 meses. Ainda permanecem desconhecidos os fatores que determinam a taxa de infecção vertical. Dados do estudo multicêntrico europeu sugerem que não haja relação entre transmissão vertical e etnia, idade materna e/ou paridade; porém, foi sugerido ser significativamente maior em mães com contagem de linfócitos CD4 inferior a 700/mm3, com relação CD4/CD8 inferior a 0.6 , e com antigenemia $\mathrm{p} 24$ persistentemente positiva (European Collaborative Study, 1992), e menor com o uso de antiretrovirais durante a gestação (Connor et al., 1994). Estes dados relacionam diretamente o maior risco de transmissão com a gravidade de doença materna durante a gestação.

\section{Infecção Intra-Útero}

Ainda não está claramente definida a época em que ocorre a transmissão materno-fetal. Existem diversas evidências de que a infecção fetal possa ocorrer in utero, como por exemplo o isolamento do HIV em tecidos de fetos de 20 (Jovaisas et al., 1985), 15 (Sprecher et al., 1986), ou até mesmo oito semanas de gestação (Lewis et al., 1990). O HIV já foi isolado a partir do cérebro, timo, baço, fígado e pulmões fetais (Kashkin et al., 1988; Rubinstein, 1986; Soeiro et al., 1992), do tecido placentário (Hill et al., 1987), e dos linfócitos presentes no sangue do cordão umbilical (DiMaria et al., 1986). Os receptores CD4 (principal alvo do HIV, presente em diversos tipos celulares principalmente linfócitos e macrófagos) de linfócitos fetais começam a surgir ao final do primeiro trimestre de gestação, o que excluiria a possibilidade de contágio embrionário; porém, estes receptores podem ser isolados em vilosidades corisis, a partir de nove semanas de amenorréia (Maury et al., 1988).

Algumas observações clínicas corroboram a hipótese de infecção intra-útero. Marion et al. (1986) descreveram uma embriopatia associada à infecção pelo HIV em neonatos, consistindo de uma síndrome dismórfica facial. Esta observação, no entanto, não foi confirmada por outros autores (Qazi et al., 1988; Blanche et al., 1989; Embree et al., 1989). Lactentes infectados por transmissão vertical freqüentemente apresentam sinais clínicos mais precocemente do que as crianças infectadas por via sangüínea (transfusão neonatal), e são mais propensos a 
sofrerem um retardo nas aquisições neuropsicomotores (Manche et al., 1990).

O mecanismo exato pelo qual o HIV cruza a barreira placentária ainda é desconhecido. A membrana trofoblástica, quando íntegra, é uma barreira eficaz contra a passagem de células sangüíneas maternas para o feto. Durante o parto, esta barreira altera-se com o rompimento físico do trofoblasto, havendo troca mais intensa de células entre mãe e filho. Entretanto, mesmo antes do parto, a passagem de substâncias solúveis e linfócitos tem sido descrita (Hunziker et al., 1984; Olding, 1972). Este pode ser um dos mecanismos pelos quais o feto adquire a infecção infra-útero, embora pareça ser menos expressivo do que a infecção por vírus livres.

Os vírus livres cruzam a placenta através de transporte ativo ou passivo, ou por infecção de células trofoblásticas. Algumas subpopulações destas células expressam o receptor CD4 e portanto representam alvos para o HIV (Maury et al., 1989). Os macrófagos placentários de Hofbauer, que teoricamente constituem uma defesa contra a infecção através da ingestão das partículas virais, poderiam servir de carreadores do vírus para a circulação fetal.

Considerando-se os vírus livres como as fontes principais de infecção intra-útero, ao contrário das infecções transfusionais e sexuais em que células infectadas são mais importantes (Levy, 1993), a sua neutralização poderia bloquear a transmissão. Esta hipótese foi sustentada por alguns trabalhos que correlacionaram a proteção à transmissão vertical com a presença de anticorpos anti-gp 120 (proteína do envelope viral através da qual o vírus se liga ao receptor CD4) (Goedert et al., 1989; Rossi et al., 1989). A ausência de anticorpos maternos de alta afinidade contra o domínio neutralizante primário da gp120 também parece relacionar-se a uma maior incidência de transmissão ao feto (Devash et al., 1990; Ugen et al., 1992; Rubinstein et al.; 1993). Se estes dados forem confirmados, a utilização de imunoterapia pode ser promissora para prevenir a infecção intra-útero pelo HIV.

A relação entre o momento da infecção e o grau de desenvolvimento do sistema imune fetal pode ser importante no estabelecimento de imunidade protetora tanto no feto quanto no recém-nato. Pouco se conhece sobre a capacidade de resposta imunológica do neonato ao HIV. Sabe-se que as crianças nascidas de mulheres HIV positivas são capazes de gerar respostas $\mathrm{T}$ citotóxicas específicas (Luzuriaga et al., 1991; Cheynier et al., 1992; Buseyne et al., 1993). Recentemente, foi demonstrado que $35 \%$ dos recém-nascidos de mulheres HIV positivas apresentam reatividade $\mathrm{T}$ helper específica contra vários peptídeos do envelope viral (Clerici et al., 1993). Nenhuma destas crianças foi infectada ao contrário daquelas que não apresentaram resposta. Esta abordagem pode ser útil como fator preditivo de risco e da necessidade de uma terapêutica antiviral precoce.

\section{Infecção Periparto}

Usando como modelo outras infecções virais como a hepatite $\mathrm{B}$, Herpes simplex, e Coxsac-kievírus, presumimos que a infecção pelo HIV também possa ocorrer durante o parto, através de contato com sangue e secreções maternas. O vírus já foi detectado em secreções cervicais (Vogt et al., 1986; Wofsy et al., 1986) e pode infectar o recém-nato através das mucosas ou microlesões cutâneas. Ainda não foi esclarecido se o tipo de parto, vaginal ou cesáreo, confere maior ou menor taxa de transmissão. No estudo multicêntrico realizado na Europa, a taxa de transmissão foi igual em crianças nascidas de parto natural ou cesáreo (European Collaborative Study, 1992). Porém, em um estudo comparando gemelares filhos de mães HIV-positivas, verificou-se que os que nasciam primeiro apresentavam um risco de transmissão de 50\%, atribuído ao maior contato com secreções e sangue, ao passo que nos segundos, o risco diminuía para 19\%. A diferença de risco foi menor nos casais de gêmeos nascidos de parto cesárea (Goeddert et al., 1991). Entretanto, o número de crianças estudadas ainda é pequeno para se chegar a uma conclusão sobre o risco real de transmissão, apresentado por um ou outro tipo de parto. Maiores coortes devem ser analisadas para esclarecimento deste ponto e, por enquanto, a Organização Mundial da Saúde (OMS) recomenda que a indicação do tipo de parto seja obstétrica. 


\section{Infecção pelo Aleitamento Materno}

A responsabilidade pela transmissão do HIV tem sido atribuída ao leite materno em casos nos quais as mães se infectaram no pós-parto. Acredita-se que a alta viremia decorrente da infecção recente tenha tido um papel importante na transmissão à criança (Ziegler et al., 1985; Oxtoby, 1988; Van de Perre et al., 1991; Palasanthiran et al., 1993). Em um estudo prospectivo realizado na África, de 212 mulheres HIV negativas durante o parto, 16 soroconverteram em um período médio de 16,6 meses de pós-parto. Nove crianças acompanharam a soroconversão materna, indicando, portanto, o leite como a via mais provável de infecção (European Collaborative Study,1992). Dados semelhantes foram descritos em Zâmbia (Hira et al., 1990).

No caso de mulheres soropositivas durante a gestação, ainda permanece desconhecido o papel adicional do leite materno no risco de transmissão do HIV. Nestes casos, o risco de infecção pelo aleitamento parece ser menos substancial do que nos casos de mulheres que soroconverteram no período de amamentação.

Biologicamente, a transmissão do HIV através do leite materno é possível. O vírus pode ser detectado por cultura (Thiry et al., 1985) ou por PCR no leite de mulheres soropositivas sintomáticas ou não. Van de Perre et al. (1988) conseguiram detectar anticorpos anti-HIV das classes IgG, IgM, e IgA no leite de mulheres soropositivas. Estes anticorpos, contudo, não foram detectados nos soros das mesmas mulheres, o que sugere que sua síntese tenha se processado no interior da glândula, provavelmente em resposta à presença do vírus no tecido mamário. Não se conhece o papel destes anticorpos, nem se eles determinam algum efeito protetor contra a transmissão à criança. Contudo, foi recentemente sugerido que a produção local de IgM específica com propriedades citotóxicas, neutralizantes, ou ambas, constituiria proteção contra células infectadas (Van de Perre et al.; 1993). Além disto, foi isolado um fator inibidor da ligação do HIV ao receptor CD4 do leite humano (Newburg et al., 1992).

Muitos debates têm ocorrido na tentativa de padronizar as indicações de alimentação ma- terna ou artificial (Mok, 1993; Ziegler, 1993). Contrabalançando o risco de infecção pelo HIV com os benefícios nutricionais, imunológicos e psicológicos que a amamentação pode proporcionar tanto à mãe quanto à criança, $\mathrm{a} O M S$ recomenda as seguintes diretrizes (WHO/Unicef, 1992): a) em países onde as moléstias infecciosas e a desnutrição sejam as causas primárias de mortalidade infantil, a amamentação deve ser recomendada para todas as mães, inclusive as HIV positivas, pois o risco de transmissão do vírus é menor do que o alto risco letal proporcionado por estas outras condições; b) em países onde a morbi-mortalidade na primeira infância sejam baixas, bem como a incidência de doenças infecciosas e desnutrição, indica-se o aleitamento artificial. No Brasil, casos particulares devem ser avaliados para a adoção de condutas direcionadas a cada região e situação social. Sempre que possível contraindica-se a amamentação e todos os esforços devem ser somados no intuito de fornecer uma alimentação artificial adequada. Porém, nas famílias com baixíssimas condições sócio-econômicas e péssimos padrões de higiene, em que o leite materno possa constituir a única fonte nutricional para o lactente, o risco de contrair o HIV pode ser menos relevante que o risco iminente de óbito por desnutrição, desidratação e/ou outras infecções. Nestes casos, não havendo alternativa, mantém-se a amamentação.

\section{CONCLUSÃO}

O impacto da AIDS na população pediátrica se faz sentir não apenas sobre as crianças infectadas. Os problemas socioeconômicos, físicos e psicológicos oriundos da doença dos pais afetam diretamente o bem-estar da criança, independentemente da sua situação sorológica. Deve-se ter em mente que, conforme as mulheres infectadas progredirem para doença clínica e êxito letal, o número de "órfãos da AIDS", infectados ou não, aumentará proporcionalmente. É necessário um esforço conjunto de pesquisadores, profissionais de saúde, pacientes e suas famílias, para que se consiga controlar esta epidemia. 


\section{RESUMO}

\section{ORTIGÃO, M. B. AIDS em Crianças:}

\section{Considerações Sobre a Transmissão Vertical.}

Cad. Saúde Públ., Rio de Janeiro, 11 (1): 142-148, jan/mar, 1995.

Trata-se de uma revisão sobre os aspectos epidemiológicos da síndrome de imunodeficiência adquirida em crianças e sobre a transmissão vertical do vírus da imunodeficiência humana (HIV). Discutem-se as diversas vias pelas quais o HIV pode transmitir-se de mãe para filho, abordando questões inerentes à fisiopatologia da infecção intra-útero, periparto e/ou pós-parto.

Palavras-Chave: AIDS; Infecção HIV; Crianças; Epidemiologia; Transmissão Vertical

\section{REFERÊNCIAS BIBLIOGRÁFICAS}

BLANCHE, S.; ROUZIOUX, C.; MOSCATO, G.; VEBER, F.; MAYAUX, M. J.; JACOMET, C.; TRICOIRE, J.; DEVILLE, A.; VIAL, M.; FIRTION, G.; deCREPY, A.; DOUARD, D.; ROBIN, M.; COURPOTIN, C.; CIRARU-VIGNERON, N.; Le DEIST, F. \& GRISCELLI, C., 1989. A prospective study of infants born to women seropositive for human immunodeficiency virus type 1. New England Journal of Medicine, 320: 1643-1648.

BLANCHE, S.; TARDIEU, M.; DULIEGE, A. M.; ROUZIOUX, C.; Le DEIST, F.; FUKUNAGA, K.; CANIGLIA, M.; JACOMET, C.; MESSIAH, A. \& GRISCELLI, C., 1990. Longitudinal study of 94 symptomatic infants with perinatally acquired human immunodeficiency virus infection: evidence for a bimodal expression of clinical and biological symptoms. American Journal of Diseases of Children, 144: 1210-1215.

BUSEYNE, F.; BLANCHE, S.; SCHMITT, D.; GRISCELLI, C. \& RIVIÈRE, Y., 1993. Detection of HIV-specific cell-mediated cytotoxicity in the peripheral blood from infected children. Journal of Immunology, 150: 3569-3581.

CHEYNIER, R.; LANGLADE-DEMOYEN, P.; MARESCOT, M. R.; BLANCHE, S.; BLONDIN, G.; WAIN-ROBSON, S.; GRISCELLI, C.; VILMER, E. \& PLATA, F., 1992. Cytotoxic Tlymphocyte responses in the peripheral blood of children born to human immunodeficiency virus-1-infected mothers. European Journal of Immunology, 22: 2211-2217.
CLERICI, M.; SISON, A. V.; BERZOFSKY, J. A.; RAKUSAN, T. A.; BRANDT, C. D.; ELLAURIE, M. L. V.; COLIE, C.; VENZON, D. J.; SEVER, J. L. \& SHEARER, G. M., 1993. Cellular immune factors associated with mother-to-infant transmision of HIV. AIDS, 7: 1427-1433.

CONNOR, E. M.; SPERLING, R. S.; GELBER, R.; KISELEV, P.; SCOTT, G.; O'SULLIVAN, M. J.; VAN DYKE, R.; BEY, M.; SHEARER, W.; JACOBSON, R. L.; AMENEZ, E.; O'WEIL, E.; BAZIN, B.; DELFARISSY, J. F.; CULNANE, M.; COOMBS, R.; ELKINS, M.; MOYE, J.; STRATTON, P. \& BALSEY, J., 1994. Reduction of maternal-infant transmission of human immunodeficiency vírus type 1 with zidovudine treatment. New England Journal of Medicine, 331: 1173-1180.

DEVASH, Y.; CALVELLI, T. A.; WOOD, D. G.; RUBINSTEIN, A., 1990. Vertical transmission of human immunodeficiency virus is correlated with the absence of high-affinity/avidity maternal antibodies to the gp120 principal neutralizing domain. Proceedings of the Nafonal Academy of Science, 87: 3445-3449.

DiMARIA, H.; COURPOTIN, C.; ROUZIOUX, C.; COHEN, D.; RIO, D.; BOUSSIN, F., 1986. Transplacental transmission of human immunodeficiency virus. Lancet, 2: 215-216.

EMBREE, J. E.; BRADDICK, M.; DATTA, P.; MURIÍTHÍ, J.; HOFF, C.; KREISS, J. K.; ROBERTS, P. L.; LAW, B. J.; PAMBA, H. O.; NDINYA-ACHOLA, J. O. \& PLUMMER, F. A., 1989. Lack of correlation of maternal human immunodeficiency virus infection with neonatal malformations. Pediatric Infectious Disease Journal, 8: 700-704.

EUROPEAN COLLABORATIVE STUDY, 1992. Risk factor for mother-to-child transnission of HIV 1. Lancet, 339: 1007-1012.

GOEDDERT, J. J.; MENDEZ, H.; DRUMMOND, J. E.; ROBERT-GUROFF, M.; MINKOFF, H. L.; HOLMAN, S.; STEVENS, R.; RUBINSTEIN, A.; BLATTNER, W. A.; WILLOUGHBY, A. \& LANDESMAN, S. H., 1989. Mother-to-infant transmission of human immunodeficiency virus type 1: association with prematurity or low anti-gp 120. Lancet, 2: 1351-1354.

GOEDDERT, J. J.; DULIEGE, A. M.; AMOS, C. I.; FELTON, S. \& BIGGAR, R. J., 1991. High risk of HIV-1 infection for the first-born twins. Lancet, 338: 1471-1475.

HILL, W. C.; BOLTON, V. \& CARLSON, J. R., 1987. Isolation of acquired immunodeficiency syndrome virus from the placenta. American Journal of Obstetrics and Gynecology, 157: 10-11. 
HIRA, S. K.; MANGROLA, U. G.; MWALE, C.; CHINTU, C.; TEMBO, G.; BRADY, W. E. \& PERINF, P. L., 1990. Apparent vertical transmission of human immunodeficiency virus type 1 by breast-feeding in Zambia. Journal of Pediatrics, 117: 421-424.

HUNZIKER, R. D.; GAMBEL, P.; \& WEGMANN, T. G., 1984. Placenta as a selective barrier to cellular traffic. Journal of Immunology, 133: 648-659.

ITALIAN MULTICENTRIC STUDY, 1988. Epidemiology, clinical features and prognostic factors of paediatric HIV infection. Lancet, 2: 1043-1046.

JOVAISAS, E.; KOCH, M. A.; SCHAFER, A.; STAUBER, M. \& LOWENTHAL, D., 1985. LAV $\backslash$ HTLV III in 20 week fetus. Lancet, 2: 1129.

KASHKIN, J. M.; SHLIOZBERG, J.; LYMAN, W. D.; CALVELLI, T. A.; STEINHAUER, E. \& RUBINSTEIN, A., 1988. Detection of human immunodeficiency virus in human fetal tissues. Pediatric Research, 23 (part 2): 355A.

KOZINETZ, C. A.; CRANE, M. F. \& REVERS, R. R., 1990. Pediatric HIV infection and AIDS: epidemiology. Seminars of Pediatric Infectious Diseases, 1: 06-16.

LEVY, J. A., 1993. Pathogenesis of human immunodeficiency virus infection. Microbiology Reviews, 57: 183-289.

LEWIS, S. H.; REYNOLDS-KOHLER, C.; FOX, H. E. \& NELSON, J. A., 1990. HIV 1 in trophoblastic and villous Hofbauer cells and haematological precursors in eight-week fetuses. Lancet, 335: 565-568.

LUZURIAGA, K.; KOUP, R. A.; PIKORA, C. A.; BRETTLER, D. B. \& SULLIVAN, 1991. Deficient human immunodeficiency virus type 1-specific cytotoxic T-cell responses in vertically infected children. Journal of Pediatrics, 119: 230-236.

MARION, R. W.; WIZNIA, A. A.; HUTCHEON, R. G.; RUBINSTEIN, A., 1986. Human T-cell lymphotropic virus type III embryopathy. American Journal of Diseases of Children, 140: 638640.

MAURY, W.; POTTS, B. J. \& RABSON, A. B., 1988. HIV Infection of CD4+, Fetal-Derived Placental Tissue. IV International Conference on AIDS, Abstracts, Stockohri, 132544.

1989. HIV 1 infection of first trimester and term human placental tissue: a possible mode of maternal-fetal transmission. Journal of Infectious Diseases, 160: 583-588.
MELBYE, M.; NJELESANI, E. K.; BAYLEY, A.; MUKELABAI, K.; MANUWELE, J. K.; BOWA, F. J.; CLAYDEN, S. A.; LEVIN, A.; BLATTNER, W. A.; WEISS, R. A.; TEDDER, R. \& BIGGAR, R. J., 1986. Evidence of heterosexual transmission and clinical manifestations of human immunodeficiency virus infection and related conditions in Lusaka, Zambia. Lancet, 2: 1113-1115.

MOK, J., 1993. Breast milk and HIV-1 transmission. Lancet, 341: 930-931.

MS (Ministério da Saúde), 1987. Programa Nacional de Doenças Sexualmente Transmissíveis/AIDS. AIDS: Boletim Epidemiológico, 1: 06.

1994. Programa Nacional de Doenças Sexualmente Transmissíveis/AIDS. AIDS: Boletim Epidemiológico, 7: 07-09.

OLDING, L. B., 1972. The possibility of materno-fetal transfer of lymphocytes in man. Acta Paediapatologica Scandinava, 61: 73-75.

OXTOBY, M. J., 1988. Human immunodeficiency virus and other viruses in human milk: placing the issues in a broader perspective. Pediatric Infectious Disease Journal, 7: 825-833.

PALASANTHIRAN, P.; ZIEGLER, J. B.; STEWART, G. J.; STUCKEY, M.; ARMSTRONG, J. A.; COOPER, D. A.; PENNY, R. \& GOLD, J., 1993. Breast-feeding during primary maternal human immunodeficiency virus infection and risk of transmission from mother to infant. Journal of Infectious Diseases, 167: 441-444.

PAPE, J. W.; LIAUTAUD, B.; THOMAS, F.; MATHURIN, J. R.; ST. AMAND, M. M.; BONCY, M.; PEAN, V.; PAMPHILE, M.; LAROCHE, A. C. \& JOHNSON, W. D., 1983. Characteristics of the acquired immunodeficiency syndrome in Haiti. New England Journal of Medicine, 309: 945-950.

QAZI, Q. H.; SHEIKH, T. M., FIKRIG, S. \& MENIKOFF, H., 1988. Lack of evidence for cranial-facial dysmorphism in perinatal human immunodeficiency virus infection. Journal of Pediatrics, 112: 07-11.

QUINN, T. C.; RUFF, A. \& HALSEY, N., 1992. Pediatric acquired immunodeficiency syndrome: special considerations for developing nations. Pediatric Infectious Disease Journal, 11: 558-568.

ROSSI, P.; MOSCHESE, V.; BROLIDEN, P. A.; FUNDARÓ, C.; QUINTI, I.; PLEBANI, A.; GLAQUITO, G.; TOVO, P. A.; LJUNGGREN, K.; ROSEN, J.; WIGZELLI, H.; JONDALL, M. $\&$ WAHREN, B., 1989. Presence of maternal antibodies to human immunodeficiency virus 1 envelope glycoprotein gp120 epitopes correlates 
with the uninfected status of children born to seropositive mothers. Proceedings of the National Academy of Science, 86: 8055-8058.

RUBINSTEIN, A., 1986. Pediatric AIDS. Current Problems in Pediatrics, 16: 361-409.

RUBINSTEIN, A.; GOLDSTEIN, H.; CALVELLI, T.; DEVAM, Y.; RUBINSTEIN, R.; SOEIRO, R. \& LYMAN, W., 1993. Materno-fetal transmission of human immunodeficiency virus-1: the role of antibodies to the V3 primary neutralizing domain. Pediatric Research, 33: S76-S79.

RYDER, R. W.; NSA, W.; HASSIG, S. E.; BEHETS, F.; RAYFIELD, M.; EKUNGOLA, B.; NELSON, A. M.; MULENDA, U. \& FRANCIS, H., 1989. Perinatal transmission of the human immunodeficiency virus type 1 to infant of seropositive women in Zaire. New England Journal of Medicine, 320: 1637-1642.

SOEIRO, R.; RUBINSTEIN, A.; RASHBAUM, W. K. \& LYMAN, W. D., 1992. Materno-fetal transmission of AIDS: frequency of human immunodeficiency virus type 1 nucleic acid sequences in human fetal DNA. Journal of Infectious Diseases, 166: 699-703.

SPRECHER, S.; SOUMENKOFF, G.; PUISSANT, F. \& DEGUELDRE, M., 1986. Vertical transmission of HIV in 15-week fetus. Lancet, 2 : 288-289.

THIRY, L.; SPRECHER-GOLDBERGER, S.; JONCKHEER, T.; LEVY, J.; VAN DE PERRRE, P.; HENRIVAUX, P.; COGNIAUX-LeCLERC, J. \& CLUMECK, N., 1985. Isolation of AIDS virus from cell-free breast milk of three healthy vitus carriers. Lancet, 2: 891-892.

UGEN, K. E.; GOEDERT, J. J.; BOYER, J.; REFAELI, Y.; FRANK, I.; WILLIAMS, W. V.; WILLOUGHBY, A.; LANDESMAN, S.; MENDEZ, H.; RUBINSTEIN, A.; KIEBER-EMMONS, T.; WEINER, D. B.; MWANDAGALIRWA, K.; DAVACHI, F.; ROGERS, M.; NZILAMBI, N.; GREENBERG, A.; MANN, J.; QUINN, T. C.; PIOT, P. \& CURRAN, J. W., 1992. Vertical transmission of human immunodeficiency virus (HIV) infection: reactivity of maternal sera with glycoprotein 120 and 41 peptides from HIV type 1. Journal of Clinical Investigation, 89: 1923-1930.
VAN de PERRE, P.; HTTIMANA, D. G. \& LEPAGE, P., 1988. Human immunodeficiency antibodies of $\operatorname{IgG}, \operatorname{IgA}$, and $\operatorname{IgM}$ subclasses in milk of seropositive mothers. Journal of Pediatrics, 113: 1039-1041.

VAN de PERRE, P.; SIMONON, A. \& HITIMANA, D. G., 1993. Infective and anti-infective properties of breastmilk from HIV-1-infected women. Lancet, 341: 914-918.

VAN de PERRE, P.; SIMONON, A.; MSELATTI, P.; HITIMANA, D. G.; VAIRA, D.; BAZUBAGIRA, A.; VAN GOETHEM, C.; STEVENS, A. M.; KARITA, E.; SONDAG-THULL, D.; DABIS, F. \& LEPAGE, P., 1991. Postnatal transmission of human immunodeficiency virus type 1 from mother to infant: a prospective cohort study in Kigali, Rwanda. New England Journal of Medicine, 325: 593-598.

VOGT, M. W.; WITT, D. J.; CRAVEN, D. E.; BYINGTON, R.; CRAWFORD, D. F.; JCHOOLEY, R. T. \& HIRSCH, M. S., 1986. Isolation of HTLV III/LAV from cervical secretions of women at risk for AIDS. Lancet, 1: 525-527.

WHO/UNICEF (World Health Organization/United Nations Children's Fund), 1992. Consensus Statement from the WHO/UNICEF. Consultation on HIV Transmission and Breastfeeding, 1992. Weekly Epidemiological Record, 24: 177-179.

WOFSY, C.; COHEN, J.; HAUER, L. B.; PADIAN, N.; MICHAELIS, B.; EVANS, L. \& LEVY, J. A., 1986. Isolation of AIDS - associated retrovirus from genital secretions of women with antibodies to the virus. Lancet, 1: 527-529.

ZIEGLER, J. B.; COOPER, D. A., JOHNSON, R. O. \& GOLD, 1., 1985. Postnatal transmission of AIDS - associated retrovírus from mother to infant. Lancet, 1: 89689.

ZIELGLER, J. B., 1993. Breast feeding and HIV. Lancet, 342: 1437-1438. 